\title{
Intermittent Chaos Characteristics Of Two Coupled Chaotic Oscillator And Its Application In The Detection Of Weak Signal 's Frequency
}

\author{
Zhao Hua \\ Career Technical College \\ Hebei Normal University \\ Hebei Shijiazhuang, 050024, China \\ zhaohualunwen@126.com
}

Li Shaohua

Department of respiration

The first hospital of Hebei Medical University

Hebei Shijiazhuang, 050031, China

1051388359@qq.com

\author{
Lv Qing \\ Career Technical College \\ Hebei Normal University \\ Hebei Shijiazhuang, 050024, China \\ The corresponding author: 1vqing1017@163.com \\ Jin Huilong \\ Career Technical College \\ Hebei Normal University \\ Hebei Normal University \\ 13131145063@163.com
}

\begin{abstract}
The detection of intermittent chaos is an important embranchment of detection of weak signal based on chaos oscillator, which is used to detect the frequency of the signal. In this paper, a method of intermittent chaos detection based on the two-oscillator-difference is put forward. The validity of this method is proved by the computer simulation test. By using this method, the range of $|\Delta \omega / \omega|$ which can be detected by intermittent chaos is extended.
\end{abstract}

Keywords-chaos; Duffing oscillator; intermittent chaos; Coupled Chaotic Oscillator; Weak signal detection

\section{INTRODUCTION}

In the 90's of 20th century, the method of detection of week signal by using chaos oscillator is proposed by Donald L.Birx ${ }^{[1]}$. Thereby, the detection of week signal that inundated in the powerful noise becomes probability, and a new application of chaos oscillator in engineering is inaugurated ${ }^{[2]}$.

In the field of signal detection using chaos, the Duffing oscillator is one of the oscillators which are studied sufficiently. The intermittent chaos appearance can be used to detect the frequency of the signal which has a little deference in frequency with the driving power of the chaos oscillator. When the absolute value of the deference is less then $3 \%$ of the frequency of the driving power, the intermittent chaos is clear ${ }^{[3]}$. By detecting the frequency of the intermittent chaos, the frequency of the week signal can be detected.

When the absolute value of the deference is more then $3 \%$ of the frequency of the driving power, the appearance of intermittent chaos also exist, but the frequency of the alternation of the condition of the phase space is high ${ }^{[4]}$. It is hard to detect a high frequency intermittent chaos by now available methods.

A method of detection of intermittent chaos is put forward by reference [5], and the frequency of the signal can be calculated by using this method. But this method relies on the filtering result of the $\mathrm{x}$ output of the chaos oscillator. In the condition of high frequency intermittent chaos, the filter also can't show the intermittent chaos clearly, so it can't breakthrough the limit that the deference of the frequency of the signal and the driving power may less then $3 \%$ of the driving power.

In this paper, a new method of intermittent chaos detection based on two duffing oscillator deference is put forward. By using this method, the absolute value of deference of frequency of the signal and the driving power, which can be detected, can be increased to $8 \%$ of the driving power. The principle of the method is expatiated, and the result of the digital simulation test is put forward

\section{DeteCtion AND FREQUENCY CALCULATION Of THE INTERMITTENT CHAOS BASED ON TWO DUFFING OSCILLATOR DEFERENCE}

\section{A. The Principle Of Intermittent Chaos}

The intermittent chaos is a particular kinetics form in which order and disorder arise by turns ${ }^{[6]}$. For Duffing oscillator, the intermittent chaos is caused by the periodical ebb and flow of the driving power. The state equation of Holmes mode Duffing oscillator is as follows:

$$
\left\{\begin{array}{l}
\frac{d x}{d t}=\mathrm{y} \\
\frac{d \mathrm{y}}{d t}=-\mathrm{k} \cdot \mathrm{y}+x-x^{3}+A(t)
\end{array}\right.
$$

In the equation above:

$$
A(\mathrm{t})=\gamma_{\mathrm{d}} \cos (t)+a \cos ((1+\Delta \omega) \cdot t+\varphi)=\gamma(t) \cos (t+\theta(t))
$$


And in it:

$$
\begin{aligned}
& \gamma(t)=\sqrt{\gamma_{a}^{2}+2 \gamma_{a} a \cos (\Delta \omega \cdot t+\varphi)+a^{2}} \\
& \theta(t)=\operatorname{arctg} \frac{a \sin (\Delta \omega \cdot t+\varphi)}{\gamma_{a}+a \cos (\Delta \omega \cdot t+\varphi)}
\end{aligned}
$$

The $\gamma_{\mathrm{d}} \cos (t)$ is the driving power of the system (it also be called the reference signal); $\gamma_{\mathrm{d}}$ is the amplitude of the driving power, its value is more or less then the critical value $\gamma_{c}$; a is the amplitude of the signal.

Form (2) equation, because of the $\Delta \omega$, the amplitude of the $A(t)$ may change from $\gamma_{\mathrm{d}}+a$ to $\gamma_{\mathrm{d}}-a$. The Duffing oscillator may sometimes go into periodic and sometimes go into chaos, which present an intermittent chaos, and its period is $\mathrm{T}=2 \pi / \Delta \omega^{[7]}$.

If we detect the period of intermittent chaos, the $\Delta \omega$ can be got, then the detection of frequency of the signal can be realized. For a is very little, the affection of $\theta(t)$ can be overlooked.

When $\omega$ is other value, it can be changed to $\omega=1$ state by linear transformation, the property of the system is unchangeable, but only the phase velocity of the system becomes $\omega$ times of primary ${ }^{[3]}$. In the situation of using single oscillator, a clear intermittent chaos needs a condition that the $|\Delta \omega / \omega|<0.03^{[7]}$.

\section{B. The Characteristic Of Output Wave Of Two Chaos Oscillator Difference}

When the Duffing oscillator is in the period motion, its $x$ output can't change tempestuously when the driving power has a little change; but when the Duffing oscillator is in the chaos motion, its $x$ output has randomicity, a little change of driving power may cause a great change of the state of the system. So, the difference of two Duffing oscillator which their reference signal amplitude have little difference can repress the periodic motion and give prominence to the chaos motion.

Based on the analysis above, we confirm two Duffing oscillator system which has oscillator $\mathrm{A}$ and oscillator B as follows:

$$
\begin{aligned}
& \text { A: }(1 / \omega)^{2}-(0.5 / \omega) \&-x+x^{3}=\gamma_{\mathrm{d}} \cos (\omega t) \ldots \ldots \ldots \\
& \text { B: }(1 / \omega)^{2}(0.5 / \omega) \&-x+x^{3}=1.001 \cdot \gamma_{\mathrm{d}} \cos (\omega t) \ldots
\end{aligned}
$$



(a) $x_{\mathrm{A} \_}$waves



(b) $x_{\mathrm{A}}-x_{\mathrm{B} \_}$waves
Figure 1. $x_{\mathrm{A} \_}$waves and $x_{\mathrm{A}}-x_{\mathrm{B} \_}$waves of two oscillator system

Solute the equation (3) and (4) by using 4 ranks Runge-Kutta method, and then make difference of the $x$ waves. When two oscillators come to periodic motion, their $x$ waves' difference is not very distinct, so the $x_{\mathrm{A}}-x_{\mathrm{B}}$ wave may be close to zero; when one or more oscillator come to chaos motion, the $x_{\mathrm{A}}-x_{\mathrm{B}}$ wave may fluctuate tempestuously because of the randomicity of the chaos motion. The $x_{\mathrm{A}}$ waves and $x_{\mathrm{A}}-x_{\mathrm{B}}$ waves of the two oscillator system are shown in Fig .1.

So the characteristic of output wave of two chaos oscillator difference is that the difference wave can repress the periodic motion and give prominence to the chaos motion.

When driving force of the oscillator $\mathrm{A}$ is set $\gamma_{d}=0.8259$, oscillator $A$ is in chaotic state, the driving force of oscillator B is $1.001 \cdot \gamma_{d}=0.8267$, so $B$ is

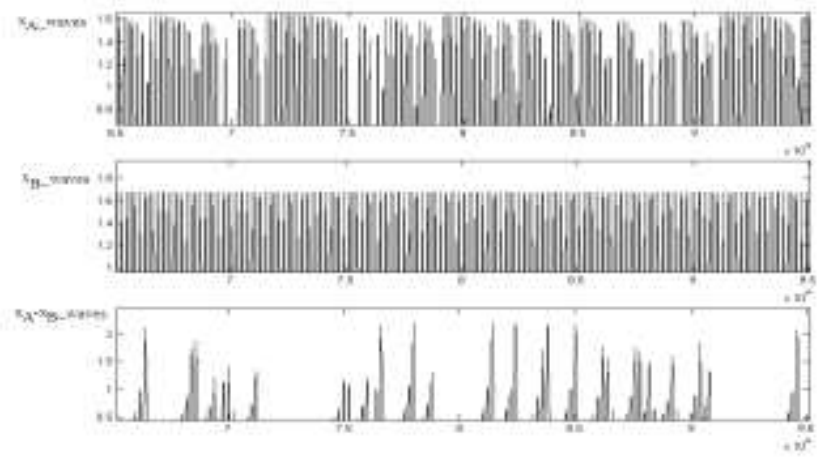

Figure 2. Coupled oscillators' motion when $\gamma_{\mathrm{d}}=0.8259$

in periodic. The coupled oscillators' motion is shown in Fig. 2.

When driving force of the oscillator $A$ is set



Figure 3. Coupled oscillators' motion when $\gamma_{\mathrm{d}}=0.8260$

$\gamma_{\mathrm{d}}=0.8260$, oscillator $\mathrm{A}$ is in chaotic state, the driving force of oscillator $\mathrm{B}$ is $1.001 \cdot \gamma_{\mathrm{d}}=0.8268$, so $\mathrm{B}$ is in periodic. The coupled oscillators' motion is shown in Fig.3.

When driving force of the oscillator $A$ is set $\gamma_{\mathrm{d}}=0.8250$, oscillator $\mathrm{A}$ is in chaotic state, the driving force of oscillator $\mathrm{B}$ is $1.001 \cdot \gamma_{\mathrm{d}}=0.8258$, so $\mathrm{B}$ is also in chaotic. The coupled oscillators' motion is shown in Fig.4. 




Figure 4. Coupled oscillators' motion when $\gamma \mathrm{d}=0.8250$

When driving force of the oscillator $\mathrm{A}$ is set $\gamma_{\mathrm{d}}=0.8258$, oscillator $\mathrm{A}$ is in chaotic state, the driving force of oscillator B is set $1.005 \cdot \gamma_{\mathrm{d}}=0.8258$, so the oscillator B is in periodic. The coupled oscillators' motion is shown in Fig .5.

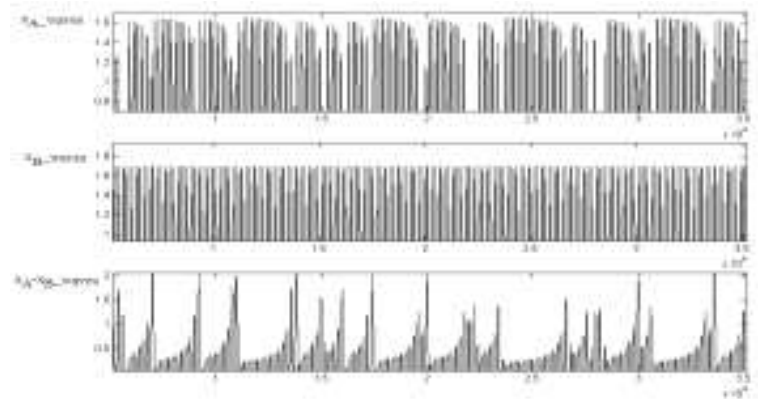

Figure 5. Coupled oscillators' motion when driving force difference is $5 \%$

\section{C. the Detection Of The Intermittent Chaos And Calculation Of Its periods}

Intermittent chaos is mainly used to detect the frequency of the signal which has little difference with the driving power in frequency ${ }^{[8]}$. An intermittent chaos detection model based on equation (3) and (4) is as follows:

$$
\begin{aligned}
& (1 / \omega)^{2}-(0.5 / \omega) \&-\mathrm{x}+\mathrm{x}^{3}=\gamma_{\mathrm{d}} \cos (\omega t)+a \cos ((\omega+\Delta \omega) t+\varphi)+\text { noise } \ldots \ldots . .(5) \\
& \text { B: }(1 / \omega)^{2}-(0.5 / \omega) \&-\mathrm{x}+\mathrm{x}^{3}=1.001 \cdot \gamma_{\mathrm{d}} \cos (\omega t)+a \cos ((\omega+\Delta \omega) t+\varphi)+\text { noise }
\end{aligned}
$$

For convenience, in the mode, $\varphi=0$, noise is Gaussian white noise with $\mu=0,|\Delta \omega / \omega|=0.015$. The $x_{\mathrm{A}}$ waves and $x_{\mathrm{A}}-x_{\mathrm{B}}$ waves of intermittent chaos are shown in Fig .6. It is obvious that the difference wave can show the intermittent chaos motion clearly.



(a) $x_{\mathrm{A} \_ \text {waves }}$

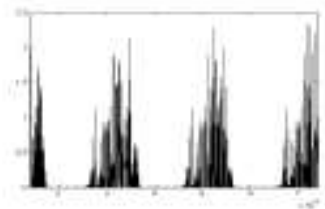

(b) $x_{\mathrm{A}-x_{\mathrm{B}}-\text { waves }}$

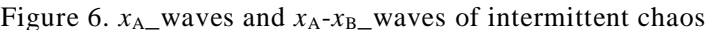

When $|\Delta \omega / \omega|>0.03$, the intermittent chaos motion can't be shown clearly by $x$ wave ${ }^{[8-10]}$. The reason is that in a single oscillator, the alternate change of periodic state and chaos state is high frequency, and the interim process between this two state affects the representation of $x$ wave of single oscillator.

Not only the interim process but also the common modulus interference can also be repressed by using the two oscillator difference system, and the real chaos motion can stand out. The intermittent chaos can be shown clearly by using this new method, and it can breakthrough the limit that $\Delta \omega / \omega \mid<0.03$.

The $x_{\mathrm{A}}-x_{\mathrm{B}_{-}}$waves when $|\Delta \omega / \omega|=0.08\left(\gamma_{\mathrm{d}}=0.826\right.$, $a=0.04)$ is shown in Fig .7.

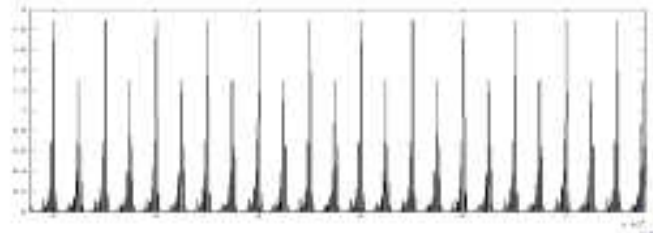

Figure 7. $x_{\mathrm{A}}-x_{\mathrm{B}} \_$waves when $|\Delta \omega / \omega|=0.08$

Seeing about Fig .6 and Fig .7, the difference wave may be divided to seriate zero area and chaos draw area, correspond to the period motion and the chaos motion in the single oscillator. The chaos draw area reflects control of chaos by the Duffing oscillator, the intensity of the draw reflects the depth of the chaos, the polarity of the draw corresponds to the focus $( \pm 1,0)$ of the oscillator.

The period of the intermittent chaos can be got by count of the seriate zero: note the start time of seriate zero as $t_{1}$ and the end time of seriate zero as $t_{2}$, then note the interim time $t_{01}=\left(t_{1}+t_{2}\right) / 2$. Thus, note the next interim time $t_{02}$, and $t_{02}-t_{01}$ is the period of the intermittent chaos.

When $|\Delta \omega / \omega|>0.05$, the seriate zero area becomes narrow and fluctuant, then the threshold of detection may be drived up.

Coupled oscillators system can restrain the periodic part in phase space, but highlight the chaotic part. Intermittent chaos of the system can be regularly displayed more clearly, so as to be able to break the bottleneck of the $\Delta \omega / \omega<0.03$ which in the single chaotic oscillator intermittent chaos detection. By using this method, the range of $|\Delta \omega / \omega|$ can be extended to $0 \sim 0.09$.

\section{Digital Simulation Test}

The method identifying the intermittent chaos and calculating its period described in section 1 is verified by using the Matlab/Simulink software to simulate the system. The simulation model is shown in Fig .8. 


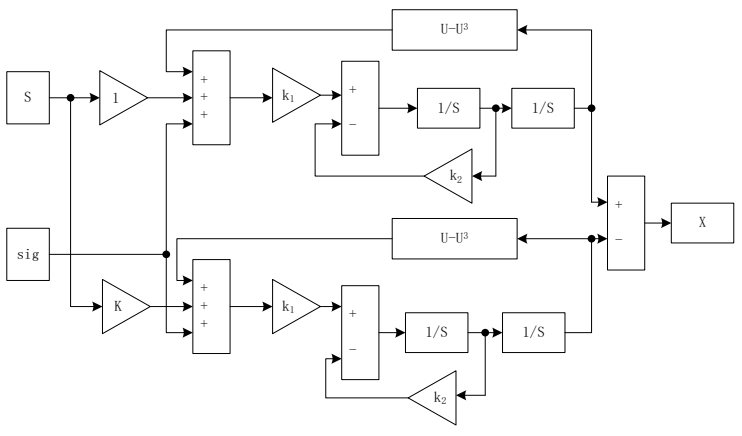

Figure 8. Two Duffing oscillator system simulation model

Using the simulation model which is shown in Fig .8, simulation experiments take $\omega=1000 \pi, \Delta \omega / \omega=0.08$, for the sake of convenience, take $\phi=0$, noise is Gauss white noise with mean is 0 and variance is 0.1 . The intermittent chaotic waves in simulation test is as shown in Fig .9 which can still clearly show the intermittent chaos phenomenon.

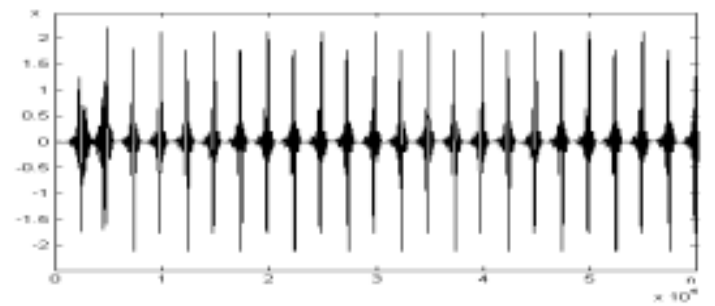

Figure 9. $\mathrm{x}_{1}-\mathrm{x}_{2}$ w waves of intermittent chaotic

The parameters of the simulation model are defined as: Reference signal amplitude $\gamma_{\mathrm{d}}=0.826$, slightly more than $\gamma_{\mathrm{c}}\left(\gamma_{\mathrm{c}}=825\right)$, frequency $f=50.9 \mathrm{~Hz} \quad(\omega=319.8 \mathrm{rad} / \mathrm{s}), \mathrm{k}=1.001$, $\mathrm{k}_{1}=\mathrm{w}^{2}, \mathrm{k}_{2}=0.5 \mathrm{w}$, the simulation step $h=0.0001 \mathrm{~s}$, simulation time $t=100 \mathrm{~s}$; The to-be-detected signal is: $\mathrm{s}(\mathrm{t})=0.04 \mathrm{sin}$ $(2 \pi f t)+\mathrm{n}(t)$, where, $\mathrm{n}(\mathrm{t})$ is the Gaussian white noise with $\mu=0$, and the target signal frequencies are 50.9, 45, 56, 46.8, 50, $54.5 \mathrm{~Hz}$, respectively

By the simulation, the following results have been obtained:

(1) In the case of the signal frequency $f=50.9 \mathrm{~Hz}$, this method estimates that the system is in great periodic motion.

(2) In the case of the signal frequency $f=45 \mathrm{~Hz}$ or $56 \mathrm{~Hz}$, this method estimates that the system is in the chaotic motion.

(3) In the case of the signal frequency $f=46.8,50$, $54.5 \mathrm{~Hz}$ respectively, this method estimates that the systems are all in the intermittent chaotic motions.

By using this method, the frequencies of the to-be-detected signal are estimated as shown in Table I.
TABLE I. RESUlTS Of SignAL FREQUENCY DETECTION

\begin{tabular}{|c|c|c|c|}
\hline \multirow{2}{*}{ SNR } & $\begin{array}{c}\text { To-be-detected } \\
(\mathbf{H z})\end{array}$ & $\begin{array}{c}\text { Estimate } \\
\text { Results }(\mathbf{H z})\end{array}$ & Error \\
\hline \multirow{3}{*}{$-5 \mathrm{~dB}$} & 46.800 & 46.792 & $-0.017 \%$ \\
& 50.000 & 50.002 & $0.004 \%$ \\
\hline \multirow{3}{*}{$10 \mathrm{~dB}$} & 54.500 & 54.495 & $-0.009 \%$ \\
& 46.800 & 46.752 & $-0.102 \%$ \\
& 50.000 & 50.036 & $0.072 \%$ \\
$-15 \mathrm{~dB}$ & 54.500 & 54.455 & $-0.082 \%$ \\
& 46.800 & 45.936 & $-1.846 \%$ \\
& 50.000 & 49.547 & $-0.906 \%$ \\
& 54.500 & 53.415 & $-1.991 \%$ \\
\hline
\end{tabular}

\section{CONCLUSION}

This paper presents a new method based on two oscillator difference to identify the intermittent chaos and calculate its period. From the simulation results, it can be seen that this method offers a simple calculation for detecting the frequency of the unknown signal by Duffing chaotic oscillator, and it can be easily realized. By using this method, the range of $|\Delta \omega / \omega|$ can be extended to 0 0.09, when $|\Delta \omega / \omega|>0.1$, the signal may be regarded as noise by the Duffing oscillator, so the clear intermittent chaos can't be find. The capability of intermittent chaos motion of Duffing oscillator on signal detection is fully exert by using this new method.

\section{ACKNOWLEDGEMENTS}

This work was financially supported by the Hebei Province Education Office Foundation (QN2014116), Hebei Normal University Youth Foundation (L2010Q10), and Hebei Normal University Doctoral Foundation (L2012B15).

\section{REFERENCES}

[1] Donald L Birx.Chaotic oscillator and CMFFNS for signal detection in noise environments[J]., 1992, 05(01):881-888

[2] Shang Qiufeng, Yin Chengqun, Li Shilin.Study on week signal detection method based on Duffing oscillator[J].Proceedings of the CSEE, 2005, 25(2):66-70

[3] WANG Guan-yu, CHEN Da-jun, LIN Jian-ya, et al.The statis-tical characteristics of weak signal detection based on Duffingoscillator[J].Acta Electronica Sinica, 1998, 26(10):38-44

[4] LENG Yong-gang, LAI Zhi-hui, FAN Sheng-bo, et al.Largeparameter stochastic resonance of two-dimensional doffing os-cillator and its application on weak signal detection[J].ActaPhysica Sinica, 2012, 61(23):230502-1

[5] Li Shilin, Yin Chengqun, Shang Qiufeng, etc.A Method of Identifying Chaotic Nature Based on Image Recognition[J].Proceedings of the CSEE, 2003, 23(10):47-50

[6] LIUHai-bo, WU De-wei, DAI Chuan-jin, et al.A new weaksinusoidal detection method based on Duffing oscillators[J].Acta Electronica Sinica, 2013, 41(1):8-12

[7] XIANG Yang, HU Xiu-lin.Maximum likelihood estimationbased high dynamic GPS carrier tracking loop[J].Acta Elec-tronica Sinica, 2010, 38(7):1563-1567

[8] LI Yue, XU Kai, YANGBao-jun.Analysis of the geometriccharacteristic quantity of the periodic solutions of the 
chaoticoscillatorsystem and the quantitative detection of weak period-ic signal[J].Acta Physica Sinica, 2008, 57(6):3353-3358

[9] ZHAI Du-qing, LIU Chong-xin, LIU Yao, et al.Determinationof the parameters of unknown signals based on intermittentchaos[J].Acta Physica Sinica, 2010, 59(2):816-825
[10] RUI Guo-sheng, ZHANG Yang, MIAO Jun, et al.Aweak sig-nal detection method by Duffing system with the gain[J].ActaElectronica Sinica, 2012, 40(6):1269-1273 